\title{
DASHBOARDS AND VISUALISATION TOOLS FOR ENHANCING CREATIVITY IN BUSINESS MASTER STUDENTS
}

\author{
Fernando González-Ladrón-de-Guevara, Marta Fernández-Diego \\ Universitat Politècnica de València (SPAIN)
}

\begin{abstract}
Dashboards are a basic element in Data Science. Well planned dashboards help the staff of a company at all levels of the organization. They allow them to ask questions and respond them in real time. As a result, this information allows them to make appropriate decisions and facilitates innovation.

A fundamental component in the dashboards are the visualizations by means of dynamic graphic objects that can be explored. These visualizations must be analyzed dynamically so that business master students can intuitively arrive at a series of insights that bring them closer to the nature of the problems.

Learning by doing and consulting. We are going to use a dashboard about innovation elaborated by Bankinter Fundation in the Platform Google Data Analytics. The proposed teaching dynamic includes the formation of work teams of 5-7 students. The challenge start when each group pose several questions to the rest of the teams. To answer these questions the students must consult the proposed dashboard. There is a time limit to answer each question. The winner is the team that answers correctly more questions and explains the way to obtain this information. This way, students get used to dashboards and visualisation tools and start learning with a good dashboard model that prepares them to later select and design proper tools. As a further result, we have appreciated that using visualisation in teaching can increase student engagement and performance.
\end{abstract}

Keywords: Business Decisions, Visualisation Tools, Teams, Dashboards.

\section{INTRODUCTION}

\subsection{Visualization}

The dazzling present amount of data, in many formats, is difficult to decipher without the help of visual analytics. With the flood of data available from information systems, business intelligence analysts and decision-makers need to make sense out of the knowledge it contains. In fact, visual analytics can provide solutions and opportunities to resolve information overload [1].

Visualization is the process of representing abstract information and data with graphical images [2]. According to [3], Information Visualization is "a process of transforming data and information that are not inherently spatial, into a visual form allowing the user to observe and understand the information." Furthermore, Information Visualization employs techniques of interactive and non-interactive computer graphics, imaging, perception, and design. Some of the key challenges involve inventing the visual metaphors, and interactive techniques to extract knowledge and discover structure in rich and widespread datasets [3].

Over fifty percent of the human brain is dedicated to vision and visual representation [4]. Visualization exploits the human visual system to extract information from data and provides an overview of complex data sets. It identifies structure, patterns, trends, anomalies, and relationships in data to assists in identifying the areas of "interest". Accentuating certain visual patterns can quickly alert human perception of certain information and effectively help human cognition in digesting data more effectively and in turn, help people make more informed decisions [5].

\subsection{Business Information Visualization}

The sheer amount of data and analysis needed in today's business environment needs to be addressed by visual interfaces that are intuitive to use and easy to comprehend [6]. Business Information Visualization "is a process of creating appropriate computer-generated visual representations of large amount of non-geometric managerial data for human problem-solving and decision-making support" [7]. 
These visual interfaces in a business environment need to be intuitive to use and easy to comprehend. That is, according to Zhang [8], visualization allows $\mathrm{BI}$ analysts to use their natural spatial/visual abilities to determine where further exploration should be done and where action is required. The underlying data could, for example, represent abstract objects, such as profit, sales, or cost.

\subsection{Dashboards}

Visualization is used to create advanced dashboards in which large amounts of information are presented on a single screen. A dashboard or report is a type of graphical user interface which often provides at-a-glance views of key performance indicators (KPIs) relevant to a particular objective or business process. The "dashboard" is usually accessible by a web browser and is often linked to regularly updating data sources. Dashboards have become popular in recent years as uniquely powerful tools for communicating important information at a glance. Although dashboards are potentially powerful, this potential is rarely realized. The greatest display technology in the world won't solve this if you fail to use effective visual design. And if a dashboard fails to tell you precisely what you need to know in an instant, you'll never use it, even if it's filled with cute gauges, meters, and traffic lights [9].

Visual design layout of dashboards are also critical for improving perception, since layout can give clarity to navigate through information clutter [5]. There are a few key elements for designing a good dashboard application [10] [11].

1 To make the complex simple, communicates easily: tell a story. It can be accessed easily by its intended audience.

2 To minimize distractions.

3 Supporting organized business with meaning and useful data. It is recommendable "choosing your end goals before putting the design elements in place" [12].

4 It applies human visual perception to visual presentation of information.

5 The data visualisation needs to correctly reflect the data and reveal details as needed.

6 Using multiple data representations, such as secondary statistics in a dashboard display to support a metric and give it better context. It is also recommendable displaying small charts alongside numeric data to improve visualisation [13].

\subsection{Dashboards Design}

It is relevant to consider some techniques to improve the design of business analytics applications:

1 To use redundant visual representations. A digital representation may not be enough. Similarly to matchmakers, We may need an analogical representation at the same time to enhance visual data perception.

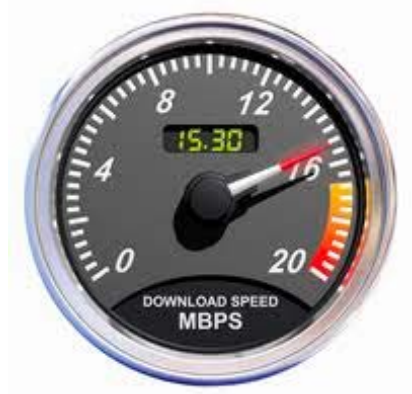

Figure 1. Analog and digital information representation.

2 Clear layout for input and output objects. It is recommendable to use dial and sliders to adjust data input. 


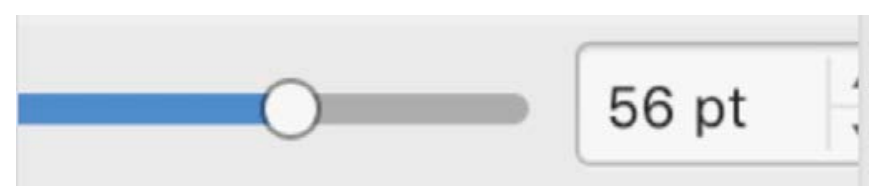

Figure 2. Slider for input data.

3 Using colors for indicators. The human eye can easily spot certain color indicators more easily and there are ways to make certain data patterns stand out. Hence, color coding is part of the visual strategy for using color effectively. Colors (hue, saturation, lightness -HSL-, and transparency) may have a positive impact on memory and attention [14]. While there are different ways to describe color digitally, HSL color model works best for designing. This model was designed in the 1920s to align color display on computer more closely with human visual perception. The aim should be to use six or fewer colors in your data visualizations. Besides, natural colors are generally better than bright or bold colors. It is also convenient to reserve bright or dark colors to highlight outliers or important calls to action. It is interesting to remember that each color in a data visualization should serve a purpose too.

4 Creating visual energy. An analytics application needs the right elements to attract audience to use them. Besides, the users will assess the rational behind the analytics model.

5 Defining the scope of the application and the range of data. Analytics applications are meant to be compact and effective. The idea of analytics dashboard is to keep it as simple and as userfriendly as possible, and not to overload data analysts with extra variables that have minimal impact on the outcome. So, don't put too many variables into it or it will be too cumbersome or complex to use, unless it is customised to for one specific user or a very few specialised users. The app will have its own limitations in terms of scope. The data variables may also have a limited range.

Business information visualization (BIV) is increasingly recognized by companies as being essential to avoid threats and realize opportunities. However, many firms still do not seem to know how to improve their BIV. Serious games appear to be a promising approach to convey this knowledge. To investigate the question whether using serious games to improve BIV skills is beneficial, they should be empirically evaluated [15].

The goal of this paper is to show how all these properties can be learnt by business master students by analysing and comparing quality dashboards that are working in business. This paper includes this introduction section, later the methodology, results, and conclusions along with the references section are presented.

\section{METHODOLOGY}

In this section, we present the tools that have been applied: Google Data Studio and the analytic tool dashboard- developed by the Observatory of the Start-up Ecosystem. Besides, the learning environment is described.

\subsection{Google Data Studio}

Google Data Studio (GDS) is a free data visualisation tool that lets to build interactive dashboards, and customized reporting. Data Studio is Google's reporting solution for power users who want to go beyond the data and dashboards of Google Analytics. Data sources include Google products (Analytics, AdWords, Search Console, Sheets, YouTube, etc.), database connectors, file uploading, and "community" connectors to popular marketing services. Google Data Studio started out as part of the enterprise Google Analytics 360 suite. In May 2016, Google announced a free version of data studio for individuals and smaller teams. At that time, there were differences between the paid version and the free version, such as the number of reports that could be created per account. By February 2017, Google announced free, unlimited Data Studio reports. Since then, the free version of Google Data Studio has continued to add powerful features and usability enhancements. In fact, Data Studio is a supercharged version of Google Analytics dashboard.

The main features and benefits of Google Data Studio are: 
- Live data connection and access to over 220 connectors that eliminate the need to schedule periodic data to refresh the reports. The dashboards are not limited in terms of what data range you can look at. It is possible to use any data range and to combine and connect multiple data sources within the same report visualising data across different datasets.

- Full control over customisation of reports and visuals, including adding as many pages or charts as needed. It is possible to customise the dashboards with company or clients colors. It is also possible to use pre-filled themes or to create a custom extracting a the from images. Besides, the layout can be edited and a wide array of charts and visuals are available.

- Dynamic controls with reports and page level filters. This feature gives significant ability to slice and dice the dataset without having to update the report setup. These dynamic controls also enable filtering through the content with dimension and date range selectors. Filters can also be used to include or exclude content.

- Drill down hierarchy makes possible to explore additional details within a chart to access more detailed information on different levels of data.

- Formulas that can include calculated metrics and fields. Additionally, the users can be given access and they have the option to customise what they're looking at without having to edit the original report.

\subsection{The Observatory of the Start-up Ecosystem}

Through the Start-ups program, the Start-ups Observatory was born with the aim of giving light to the entrepreneurial activity in Spain. Hence, its purpose is to provide a barometer on investing in start-ups in Spain that is useful to both markets and the ecosystem of entrepreneurs. To fulfil its mission of providing value to the ecosystem, the Observatory makes available to entrepreneurs, media and people interested in the entrepreneurship scene a digital tool, public and free, with data on investment in start-ups. This tool will be constantly fed with new public information and is also open to those who wish to provide the actors in the field of start-ups in order to enrich it.

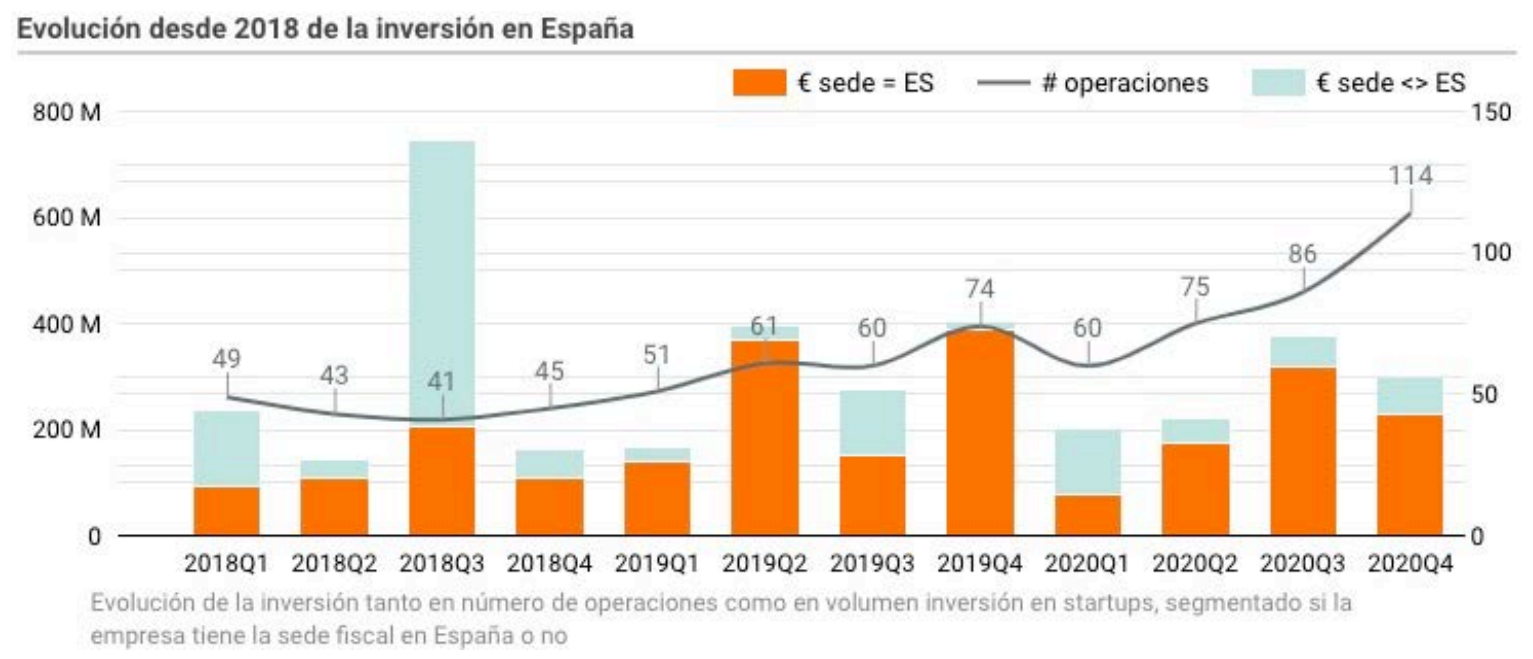

Figure 3. Dynamic graph about investment evolution in Spain

Thus, the Observatory (Observatorio Start-ups España [16]) collects all the data published from the funding rounds in start-ups in Spain since 2018, presents analysis of the main aspects of the ecosystem and allows interaction with the data, so that it can serve as a source of reference in decision making.

\subsection{Learning Environment}

This tool has been used for 15 students of the Master's Degree in Business Management, Products and Services at Universitat Politècnica de Valéncia. This master aims to complete the training of graduates so that they are able to take on the current challenges of globalization, technological progress and the Knowledge Society. These students take a course about ICT called "Senior Management and Information Technologies". One of the learning objectives of this course is the usage 
and basic design of analytic dashboards. In this regard, the students have formed teams of 5-7 students to analyze the tool proposed by the Start-up Ecosystem Observatory.

\section{RESULTS}

The students have followed a group dynamics consisting in asking questions to their peers related with the topic of the Start-up Ecosystem. These time-limited questions must be answered using the aforementioned analytics tool. This is an intuitive way to learn that has made possible for them to get used with the main features of this dashboard. As a result, the students have obtained a good understanding of the Start-up Ecosystem Dashboards. Particularly, they have dealt with information about:

- Global data on investment in Spain, with details of the main sectors, operations and most active investors.

- Interannual perspectives of the investment, comparing different data such as the size of each round.

- Analysis and investment trends, with information on monthly evolution, types of investors or origin of funds.

- Analysis of exit operations in order to anticipate trends, with data on the evolution and origin of the funds, among others.

It is also quite relevant to mention that they have taken into consideration the design of this tool. Consequently, the students have defined some convenient features for designing Data Studio reports:

- It is interesting to use the theme settings to avoid modifying individual style properties.

- Fonts selection.

- Selecting the right artifacts to present the information.

- Using a color palette. They have realized the convenience to use pick neutrals colors that are not black \& white. Using a dark grey for text, and a light grey for lines is often more effective.

- Customization of the different features of a dashboard.

- Definition of a page with a brief introduction to the tool.

- Utilizing tools for filtering and selecting a range of data.

- Using grid lines to space elements evenly.

- The use of consistent alignment for readability -maintaining consistency throughout the report by left-aligning titles, body copy, graphics, and any other elements to help readers quickly absorb the content.

These elements are the starting point for the elaboration of an assessment rubric that they should know before applying it. Regarding the students satisfaction, this gamification approach has made possible for them to get used with basic concepts and obtain competences about dashboards design.

\section{CONCLUSIONS}

This paper reflects the learning experience of 15 Business Master students at Universitat Politècnica de València in relation with digital dashboards and information visualization. It has been followed an approach of learning by comparing and benchmarking a prominent dashboard. This dashboard has been elaborated by The Observatorio of Startup Ecosystem in Spain. The learning experience has enabled the students to get used with usual digital objects and dashboard design good practices. This gamification approach based in group dynamics of questions and answers has also improved the students satisfaction.

\section{REFERENCES}

[1] J. Thomas, "Visual analytics: a grand challenge in science: turning information overload into the opportunity of the decade," J. Comput. Sci. Coll., vol. 23, no. 2, pp. 5-6, 2007. 
[2] D. P. Tegarden, "Business information visualization," Commun. Assoc. Inf. Syst., vol. 1, no. 1, p. 4, 1999.

[3] N. Gershon and S. G. Eick, "Visualization's new tack: making sense of information," IEEE Spectr., vol. 32, no. 11, pp. 38-40, Nov. 1995, doi: 10.1109/6.469330.

[4] R. Jerath and M. W. Crawford, "Layers of human brain activity: a functional model based on the default mode network and slow oscillations," Front. Hum. Neurosci., vol. 9, p. 248, 2015.

[5] A. Y. Yap, "Creating Business Analytics Dashboard Designs Using Visualization Methodologies: Case Methods for Innovative Analytics Pedagogy," Inf. Syst. Educ. J., vol. 18, no. 5, pp. 25-33, Oct. 2020.

[6] D. Keim, J. Kohlhammer, G. Ellis, and F. Mansmann, "Mastering the information age: solving problems with visual analytics," 2010.

[7] P. Zhang and A. B. Whinston, "Business information visualization for decision-making support-a research strategy," 1995.

[8] P. Zhang, "Business information visualization: guidance for research and practice," Encycl. Microcomput., vol. 27, no. Supplement 6, pp. 61-77, 2001.

[9] S. Few, Information dashboard design: The effective visual communication of data. O'Reilly Media, Inc., 2006.

[10] I. Hertz, "Dashboard Design Best Practices - 4 Key Principles," Sisense, Aug. 07, 2019. https://www.sisense.com/blog/4-design-principles-creating-better-dashboards/ (accessed Jan. 05, 2021).

[11] Analytics Demystified, "The Perfect Dashboard: Three Pieces of Information," Analytics Demystified, Nov. 09, 2009. https://analyticsdemystified.com/reporting/the-perfect-dashboardthree-pieces-of-information/ (accessed Jan. 05, 2021).

[12] S. Durcevic, "Top 20 Dashboard Design Principles, Best Practices \& How To's," BI Blog | Data Visualization \& Analytics Blog | datapine, Sep. 09, 2020. https://www.datapine.com/blog/ dashboard-design-principles-and-best-practices/ (accessed Jan. 05, 2021).

[13] B. K. Smith, "Comparing small graphs: how distance, orientation, and alignment affect the comparability of small multiple bar graphs," PhD Thesis, 2012.

[14] V. BI, "How to Effectively Use Colors in Dashboard and Report Design," Visual BI Solutions, Sep. 13, 2018. https://visualbi.com/blogs/business-intelligence/dashboards/use-colors-dashboardreport-design/ (accessed Jan. 05, 2021).

[15] C. Grund and M. Schelkle, "Developing a serious game for business information visualization," 2016.

[16] "Observatorio Startups España," Google Data Studio. http://datastudio.google.com/ reporting/ 683aa010-4b0a-435e-9d03-27e5fa07d7f6/page/oPJEB?feature=opengraph (accessed Jan. 05, 2021). 